\title{
Comparação do equilíbrio e da mobilidade funcional entre pacientes com doença de Parkinson ativos e inativos
}

\author{
Comparison of balance and functional mobility among active and \\ inactive patients with Parkinson's disease
}

\section{Paulo Cezar Rocha dos Santos; Luana Carolina de Morais; Lucas Simieli; Ellen Lirani-Silva; Rodrigo Vitório; Maria Dilail- ça Trigueiro de Oliveira Ferreira'; Maria Joana Duarte Caetano²; Lilian Teresa Bucken Gobbi}

\section{Resumo}

A doença de Parkinson compromete o equilíbrio e a mobilidade funcional. A atividade física tem um importante papel se contrapondo aos efeitos da doença, podendo promover benefícios na mobilidade de pacientes. O estudo teve como objetivo comparar o desempenho em equilíbrio e mobilidade funcional de pacientes com doença de Parkinson ativos e inativos. Participaram do estudo 41 pacientes distribuídos, em ativos (19) e inativos (22), de acordo com a pontuação obtida no questionário de Baecke modificados para idosos e confirmado pela quantidade de prática regular de atividade física. Todos os pacientes foram avaliados em relação ao equilíbrio através da escala de equilíbrio de Berg e em relação à mobilidade funcional através do Timed up and Go test. Não foram encontradas diferenças significativas em relação ao equilíbrio. Entretanto, pacientes ativos apresentaram melhores desempenhos $(6,86 \pm 1,16 \mathrm{~s})$ na mobilidade funcional quando comparado com pacientes inativos $(9,19 \pm 4,40 \mathrm{~s})$. Manter níveis ótimos de atividade física é um importante fator para a melhora da mobilidade de pacientes com doença de Parkinson, refletindo na realização das atividades diárias e na qualidade de vida.

\section{Palavras-chave}

Mobilidade funcional; Equilíbrio; Atividade física; Doença de Parkinson.

\begin{abstract}
Parkinson's disease affects the balance and mobility. Physical activity has an important role in minimizing the effects of the disease and may provide benefits in patient mobility. The study aimed to compare the balance and mobility performance of active and inactive patients with Parkinson's disease. The study included 41 patients distributed in active (19) and inactive (22) groups according to the score obtained in the Baecke questionnaire modified for the elderly and it was confirmed by amount of the practice of regular physical activity. All patients were assessed for the balance through the Berg balance scale and in relation to mobility through the Timed Up and Go test. There were no significant between group differences for balance. However, active patients were better (6.86 $\pm 1.16 \mathrm{~s}$ ) in mobility when compared with inactive patients (9.19 \pm 4.40 s). Maintaining optimal levels of physical activity is an important factor to improve the mobility performance of patients with Parkinson's disease, reflecting in daily activities and quality of life.
\end{abstract}

\section{Keywords}

Mobility; Balance; Physical Activity, Parkinson's Disease.

\section{Introdução}

A morte das células dopaminérgicas que afetam o funcionamento dos núcleos da base desencadeia a doença de Parkinson ${ }^{1}$. Com isso, diversos comprometimentos motores ocorrem ${ }^{2}$, dentre eles hipometria (diminuição na amplitude dos movimentos), bradicinesia (lentidão dos movimentos), distúrbios de equi-

1 UNESP - Universidade Estadual Paulista Júlio de Mesquita Filho - Campus Rio Claro - Departamento de Educação Física - LEPLO: Laboratório de Estudos da Postura e Locomoção. São Paulo - Brasil

2 Neuroscience Research Australia, University of New South Wales, Sydney, NSW, Australia. School of Public Health \& Community Medicine, University of New South

Wales, Sydney, NSW, Australia líbrio, rigidez muscular e tremor de repouso ${ }^{3}$, que vem a comprometer a independência da mobilidade funcional de pacientes com a doença de Parkinson ${ }^{2}$.

Comprometimento na mobilidade funcional diminui a funcionalidade e compromete a qualidade de vida de pacientes com doença de Parkinson ${ }^{4}$. $\mathrm{O}$ andar é a forma mais natural de se locomover ${ }^{5}$ e, geralmente, garante independência nas atividades da vida diária ${ }^{2}$. Estudos têm verificado que pacientes com doença de Parkinson que têm dificuldades na mobilidade fun- 
cional, mostram diminuição da velocidade do andar ${ }^{4}$. Além disso, esses pacientes têm demonstrado um alto índice de quedas ${ }^{6}$, atribuídos aos comprometimentos no equilíbrio e na locomoção. Ferramentas têm sido usadas frequentemente com a finalidade de avaliar a mobilidade funcional e o equilíbrio ${ }^{7,8} \mathrm{em}$ idosos e em pacientes com a doença de Parkinson, dentre elas o teste Timed-Up and Go (TUG) ${ }^{9,10}$ se mostra com importante validade ecológica por combinar ações sequenciais comuns do cotidiano como levantar da cadeira e andar com mudança de direção; e a escala de equilíbrio de Berg $(\mathrm{EEB})^{11}$. Especificamente envolvendo está avaliação para mobilidade funcional, pacientes com DP apresentam maior lentidão no TUG quando comparado com seus pares sem a doença ${ }^{10,12}$, além ainda de efeito positivo do medicamento na mobilidade funcional de pacientes com $\mathrm{DP}^{12}$. Enquanto que envolvendo o equilíbrio, problemas parecem estar relacionados com os comprometimentos específicos da doença ${ }^{13}$, sendo recomendado atividade física como forma de intervenção ${ }^{8}$.

A terapia medicamentosa, através da dopamina sintética, tem sido a principal forma de tratamento da doença ${ }^{14}$. Entretanto, é comprovado que o uso prolongado deste medicamento pode ocasionar discinesias ${ }^{15}$, aumentando assim as dificuldades no controle do equilíbrio e da mobilidade funcional. Associada à terapia medicamentosa, a prática regular de atividade física tem mostrado benefícios na qualidade de vida da população ${ }^{16}$ por promover melhora nos componentes da capacidade funcional ${ }^{17}$, no planejamento e execução das atividades da vida diária ${ }^{15} \mathrm{e}$ na mobilidade funcional ${ }^{5}$, além de conter o caráter progressivo da doença ${ }^{19}$.

Um indicativo da prática regular de atividade física é obtido por meio do questionário Baecke modificado para idosos ${ }^{20}$, onde é possível avaliar as atividades realizadas no âmbito doméstico, no tempo livre e nas atividades de lazer. Este instrumento tem sido utilizado em vários estudos como forma de caracterização da amostra. Entretanto, esse questionário pode auxiliar na formação de grupos de indivíduos ativos e inativos, facilitando a comparação dos desempenhos em equilíbrio e mobilidade funcional. Ainda, existe uma lacuna na literatura a respeito do desempenho em equilíbrio e mobilidade funcional de pacientes com doença de Parkinson. Sendo assim, o objetivo deste estudo foi comparar o desempenho em equilíbrio e mobilidade funcional de pacientes com doença de Parkinson ativos e inativos. Espera-se que pacientes ativos tenham melhor desempenho em equilíbrio e mobilidade funcional quando comparados com pacientes inativos.

\section{Métodos}

Todos os procedimentos experimentais foram realizados no Laboratório do Estudo da Postura e da Locomoção (LEPLO), no período de 2006 até 2008, e seguiu o seguinte delineamento: i) Seleção dos participantes de acordo com os critérios de inclusão/exclusão; ii) distribuição dos participantes em ativo e inativo, de acordo com o nível de atividade física; iii) após a distribuição, os pacientes compareceram no LEPLO em dois dias: - Dia A) Avaliação clínica ("Unified Parkinson's Disease Rating Scale" - UPDRS) ${ }^{21}$, estadiamento da DP (Hoebn ఓ Yahr - H\&Y $)^{22}$ e condição cognitiva por meio do Mini Exame do Estado Mental (MEEM) ${ }^{23}$ o cognitivo; - Dia B) Avaliação antropométrica, do Equilíbrio e da mobilidade funcional, por meio de mensuração de peso e altura, da EEB e do TUG, respectivamente.

Inicialmente foram selecionados 47 pacientes com doença de Parkinson idiopática juntamente ao banco de dados ao Programa de Atividade Física para Pacientes com Doença de Parkinson (PROPARKI), referente a avaliação inicial dos pacientes que se inscreveram no PROPARKI entre os anos de 2006 até 2010. Entre- 
tanto, 6 pacientes com DP foram excluídos por apresentarem: i) Outra desordem neurológica $(\mathrm{n}=2)$; ii) compromentimentos vestibulares, visuais e somatossensoriais que impossibilitasse os pacientes de realizar a tarefa $(\mathrm{n}=1)$; iii) pontuação inadequada (entre 4 e 6 ) para compor um dos grupos de acordo com a prática regular de atividade física pelo questionário de Baecke (sendo considerado a pontuação $\leq$ 4 inativo e $\geq 6$ ativo - $n=2$ ); iv) Estágios acima de 3 na escala de H\&Y, comprometendo assim, a mobilidade independente $(n=1)$. Sendo assim, 41 pacientes com DP cumpriram os critérios de inclusão (DP de característica idiopática, cumpriram a pontuação no questionário de Baecke e locomoção independente)

Os participantes, de ambos os sexos, foram classificados em ativos ( $\mathrm{N}=19$, sendo 10 homens e 9 mulheres) e inativos ( $\mathrm{n}=22$, dos quais são 11 homens e $11 \mathrm{mu}$ lheres) por meio da pontuação obtida no Questionário de Baecke modificado para idosos ${ }^{20}$, sendo que para integrar o grupo ativo, o paciente deveria obter pontuação igual ou superior a seis; enquanto que pontuação inferior ou igual a quatro foi empregada para compor o grupo inativo ${ }^{24,25}$. Além disso, a distribuição foi confirmada pela prática regular de atividade física de acordo com as recomendações do Colégio Americano de Medicina do Esporte ${ }^{24}$, onde foram considerados ativos os pacientes que realizavam mais que $150 \mathrm{~min}$ de atividade física distribuídos em pelo menos 3 dias por semana, avaliado por meio de anamnese.

Após a distribuição, os pacientes com DP foram convidados a comparecer no laboratório em 2 dias, ambos no estado "ON" do medicamento específico para DP (aproximadamente 1 hora após a ingestão), para garantir a condição de efeito do medicamento para todos os pacientes:

- Dia A: Os pacientes com DP, responderam uma anamnese para verificar os critérios de inclusão e exclusão e, posteriormente, foram avaliados por neuropsiquiatra, quanto ao acometimento e severidade da doença pela UPDRS ${ }^{21} \mathrm{e}$ pela escala $\mathrm{H} \& \mathrm{Y}^{22}$. Em relação às condições cognitivas, os participantes foram avaliados pelo MEEM $^{23}$. A UPDRS avalia o curso longitudinal da doença em três principais partes: 1) Psíquica: que avalia, por auto relato, características de comportamento e de humor; 2) Funcional: auto avaliação das atividades da vida diária, incluindo a fala, a deglutição, escrita, o vestir, higiene, quedas, salivando, virando na cama, andar e cortar os alimentos; 3) Motora: avaliação motora realizada por um clinico com a finalidade de verificar os sinais e sintomas da doença, assim como características de uni ou bilateralidade. O H\&Y é uma escala usada para descrever como está o progresso da doença em relação ao participante. A escala vai de 1 até 5 , sendo que de acordo com o maior comprometimento dos pacientes e a progressão da doença mais avançado ele se encontra na escala de H\&Y. Por último, avaliamos a condição cognitiva por meio do MEEM, sendo possível avaliar a cognição considerando os domínios: orientação espacial, temporal, memória imediata e de evocação, cálculo, linguagem-nomeação, repetição, compreensão, escrita e cópia de desenho.

- Dia B: Foram mensurados peso e altura dos pacientes com DP, por meio de uma régua métrica fixa na parede e de uma balança, respectivamente. Posteriormente, foi avaliado o equilíbrio por meio da $\mathrm{EEB}^{10,27,28}$. A escala possui 14 itens que mensura o equilíbrio estático e dinâmico. Cada item possui cinco possibilidades escalares de respostas de 0 - 4, sendo que 0 o participante não conseguiu realizar a tarefa referente ao item e 4 realizou de forma normal. Sendo assim, quanto maior a pontuação obtida no teste melhor o equilíbrio dos participantes. A pontuação de cada participante foi utilizada para análise. Por 
último, a mobilidade funcional foi avaliada por meio do $\mathrm{TUG}^{9}$, onde o participante, após o comando "vai", deveria levantar da cadeira andar o mais rápido a distância de 3 metros, dar a volta em um cone, voltar e sentar novamente na cadeira. O tempo de realização da tarefa foi mensurado. Cada participante realizou 3 tentativas sendo considerado o valor médio para análise.

As análises foram realizadas no software SPSS 15.0 for Windows ${ }^{\circledR}$. O critério estatístico para significância foi $\mathrm{p}<0,05$ para todas as análises. Foi verificado normalidade e homogeneidade dos dados, por meio dos testes de Shapiro-Wilk e Levene, respectivamente. Para verificar a similaridade entre os grupos, as características antropométricas de peso e altura, a idade, a UPDRS, H\&Y e o MEEM foram comparados através de ANOVA. O mesmo teste estatístico foi utilizado para comparar as pontuações da EEB e as médias das 3 tentativas do TUG entre os grupos (ativo $\mathrm{x}$ inativo).

Todos os pacientes foram devidamente informados e concordaram em participar por meio da assinatura do termo de consentimento (protocolo $\mathrm{n}$. 002529/2007), aprovado pelo comitê de ética local.

\section{Resultados}

Os grupos obtiveram médias semelhantes para idade ( $\mathrm{F}=1,48, \mathrm{p}=0,702)$, estatura $\left(\mathrm{F}_{1,39}=2,451, \mathrm{p}=1.26\right)$ e peso $\left(\mathrm{F}_{1,39}=0,044, \mathrm{p}=0,834\right)$. Da mesma forma, não foram encontradas diferenças entre grupos para variáveis de estadiamento da doença $\mathrm{H} \& \mathrm{Y}\left(\mathrm{F}_{1,39}=0,003, \mathrm{p}=0,958\right)$, do comprometimento da DP - UPDRS $\left(\mathrm{F}_{1,39}=1,254\right.$, $\mathrm{p}=0,27)$ e MEEM $\left(\mathrm{F}_{1,39}=0,049, \mathrm{p}=8,23\right)$, como apresentado na Tabela 1.

TABELA 1 - Médias e desvios padrão das variáveis de caracterização e Clínicas estadiamento da DP pela escala de Hoehn \& Yahr (H\&Y), de comprometimento pela Unified Parkinson's Disease Rating Scale (UPDRS) e condições cognitivo pelo Mini Exame do Estado Mental por Grupo.

\begin{tabular}{lcccccc}
\hline Grupo & Idade (anos) & Estatura $(\mathrm{cm})$ & Peso (kg) & H\&Y (estágio) & UPDRS (ptos) & MEEM (ptos) \\
\hline Ativo & $68,47 \pm 8,85$ & $164,39 \pm 8,26$ & $71,78 \pm 10,26$ & $1,58 \pm 0,69$ & $41,00 \pm 18,55$ & $27,53 \pm 1,98$ \\
\hline Inativo & $69,54 \pm 8,91$ & $159,18 \pm 10,43$ & $70,97 \pm 13,75$ & $1,57 \pm 0,62$ & $35,64 \pm 11,81$ & $27,36 \pm 2,63$ \\
\hline
\end{tabular}

Para a mobilidade funcional (Figura 1), a ANOVA revelou diferença significativa $\left(\mathrm{F}_{1,39}=4,980, \mathrm{p}=0,031\right)$, sendo que o grupo ativo obteve menos tempo no TUG $(6,86 \pm 1,16 \mathrm{~s})$ quando comparado com o grupo inativo $(9,19 \pm 4,40 \mathrm{~s})$.

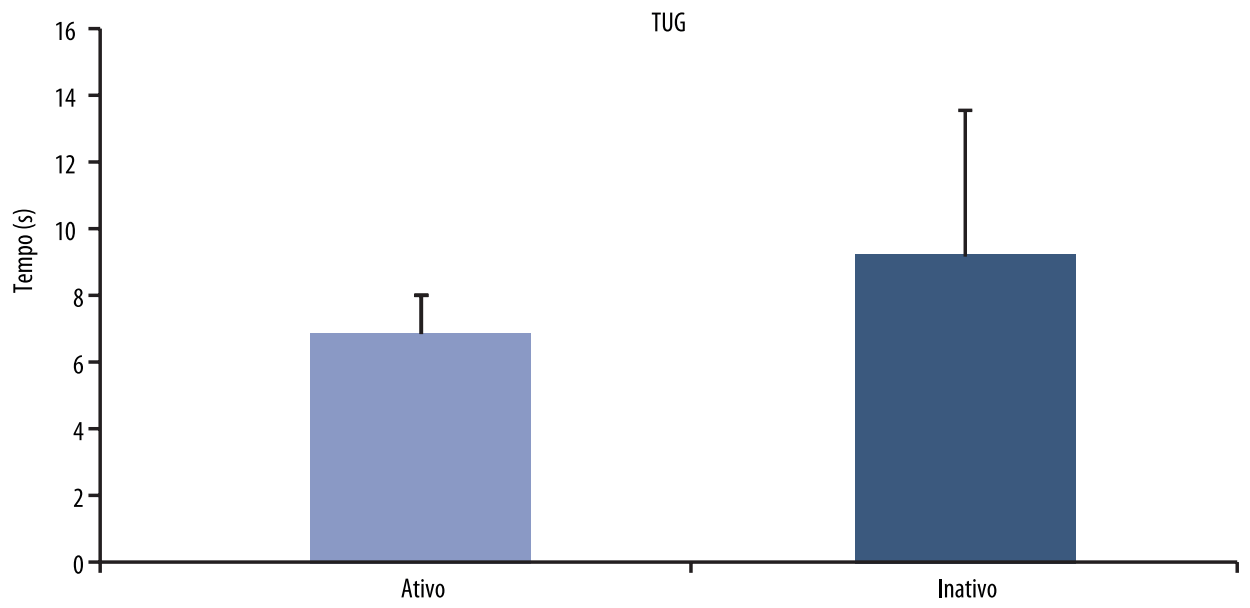

FIGURA 1 - Médias e desvios padrão do desempenho em mobilidade por grupo. 
Já para o equilíbrio (Figura 2), não foram reveladas diferenças significativas $\left(\mathrm{F}_{1,39}=2,311, \mathrm{p}=0,137\right)$, com pontuações similares na EEB entre os grupos (Ativo = $54,16 \pm 2,24$ pontos, Inativo $=52,18 \pm 5,26$ pontos $)$.

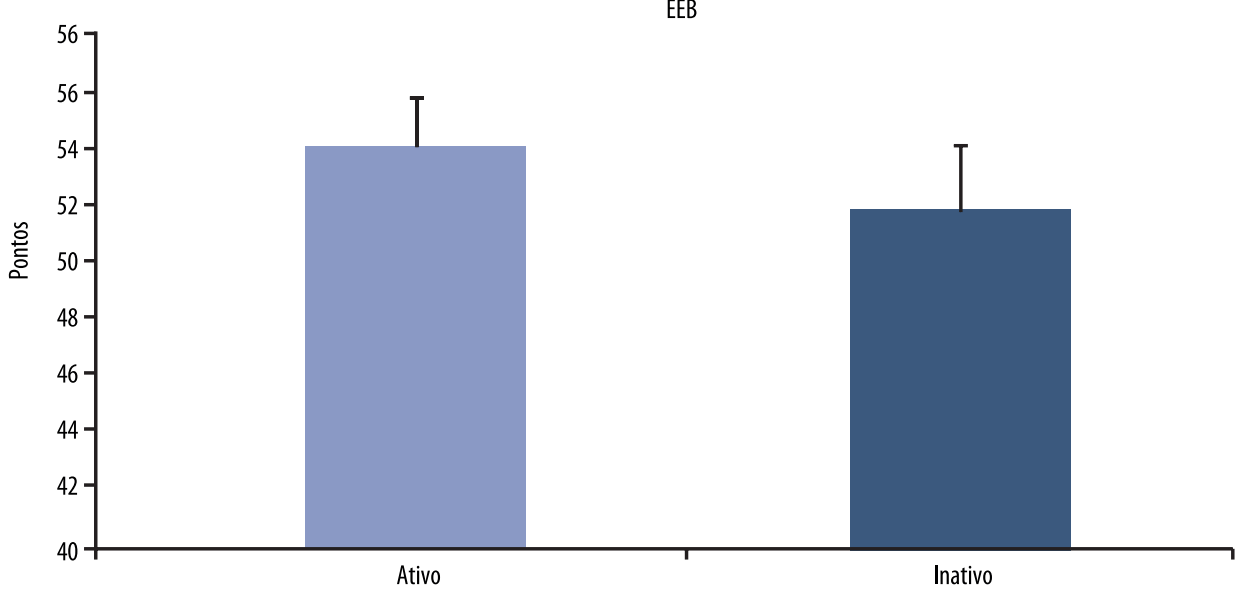

FIGURA 2 - Médias e desvios padrão do desempenho em equilíbrio por grupo.

\section{Discussão}

O objetivo deste estudo foi comparar o desempenho em mobilidade funcional e equilíbrio de pacientes com doença de Parkinson ativos e inativos. As expectativas do estudo foram parcialmente atingidas, mostrando que o nível de atividade física influencia na mobilidade funcional de pacientes com doença de Parkinson. Os pacientes ativos realizaram a tarefa do TUG em menor tempo do que pacientes inativos. Entretanto, para a tarefa de equilíbrio não houve diferença entre os grupos.

A atividade física promove melhora na mobilidade funcional de pacientes com doença de Parkinson. Pacientes com DP que realizam prática regular de atividade física apresentam menor tempo no TUG corroborando com achados na literatu$\mathrm{ra}^{29}$. Isto pode ser explicado pelos ganhos nos componentes da capacidade funcional devido à realização de atividade física regular ${ }^{27}$. Além disso, a atividade física reflete em aumento nos níveis de potência aeróbia, potência muscular, flexibilidade e estabilidade postural, que são componentes essenciais para a realização de ações motoras sequenciais, comuns nas tarefas diárias, como o sentar e levantar e a locomoção ${ }^{8}$. Sendo assim, manter boa mobilidade funcional promove benefícios nas atividades da vida diária e, consequentemente, na qualidade da vida ${ }^{17}$.

Especificamente em relação à doença de Parkinson, a prática de atividade física tem apresentado benefícios na plasticidade sináptica ${ }^{30}$, refletindo em aumento na quantidade de neurônios $^{31}$, maior eficiência dos neurotransmissores ${ }^{32}$ e aumento e melhor captação da dopamina. Diante destes benefícios, pode-se inferir que, além dos efeitos positivos nos componentes da capacidade funcional ${ }^{14}$, manter-se fisicamente ativo pode amenizar os sinais e sintomas da doença, como é o caso da bradicinesia, refletindo assim em aumento na velocidade, e maior eficácia na realização de tarefas motoras, assim como ganho nas tarefas de vida diária ${ }^{15}$ e, consequentemente, na qualidade de vida dos pacientes ${ }^{5}$. Este argumento é reforçado pelos resultados encontrados por Gobbi e colaboradores ${ }^{8}$ que verificou efeito positivo do exercício físico na mobilidade de pacientes com DP, corroborando com os resultados do presente estudo e evidenciando a relevância da prática regular de atividade física na mobilidade de pacientes com DP.

Cabe destacar também, que o desempenho na tarefa de TUG, observado pela média de tempo, para ambos os grupos, foi melhor do que o relatado na litera- 
tura $^{10,12}$. Destacam-se os valores médios de tempo do grupo de pacientes ativos abaixo dos valores encontrados para idosos sem a doença de Parkinson ${ }^{29}$. Esta observação indica que a prática regular de atividade física parece nivelar os pacientes em estágios iniciais da doença de Parkinson e os idosos sem a doença em tarefas de andar em máxima velocidade.

A EEB, apesar de amplamente usada em diferentes populações, não foi sensível para apontar diferenças entre os grupos. Tanto pacientes ativos como inativos obtiveram médias próximas do valor teto do teste e, ainda, pontuação similar aos encontrados para idosos sem a doença de Parkinson ${ }^{9}$. Esta observação indica que as tarefas propostas pelo teste não são desafiadoras para os pacientes no estágio inicial da doença. E, também podem indicar que a capacidade dos núcleos da base em integrar informações sensoriais ainda está preservada em pacientes em estágios iniciais da doença de Parkinson ${ }^{3}$, consequentemente, refletindo na capacidade preservada do controle de equilíbrio.

As evidencias apontadas no presente estudo reforçam a importância de exercícios para a funcionalidade, sendo que por meio dos resultados obtidos na mobilidade funcional indicam que este parâmetro parece ser mais sensível a atividade física generalizada ${ }^{8}$. Enquanto que o equilíbrio parece não ser tão sensível, reforçando a necessidade de estratégias de intervenção específica para o equilíbrio, como sugere Qutubuddin e colaboradores ${ }^{11}$. Além disso, algumas limitações são evidenciadas. Embora os testes utilizados no estudo serem amplamente empregados em diversos estudos e na prática clínica, há necessidade de uma análise mais minuciosa de seus procedimentos e resultados obtidos. No que se refere à mobilidade funcional e ao equilíbrio, sugere-se a análise utilizando ferramentas cinemáticas e cinéticas, que permitem verificar parâmetros espaço-temporais do andar e deslocamento do centro de gravidade. Dados mais precisos e confiáveis fornecidos por essas ferramentas possibilitariam a análise mais detalhada do comportamento motor de pacientes com doença de Parkinson. Além disso, a ausência de um grupo de idosos sem a doença não possibilita o entendimento acerca as diferenças entre os efeitos da prática regular de atividade física em idosos sem e com DP. Entretanto, os resultados podem auxiliar no direcionamento de programas de intervenção futuro, podendo se contrapor aos efeitos negativos da DP na mobilidade funcional ${ }^{10}$

Diante dos resultados apresentados, conclui-se que manter-se ativo é um importante fator para mobilidade funcional de pacientes com doença de Parkinson, com consequências na realização das atividades diárias e na qualidade de vida desses indivíduos. Em relação ao equilíbrio, tanto os indivíduos ativos como os inativos obtiveram valores próximos ao teto do teste, dificultando a indicação de diferenças relacionadas ao nível de atividade física em pacientes em estágio leve e moderado da doença de Parkinson. Assim, sugere-se a realização de estudos que consigam analisar parâmetros mais detalhados em relação à mobilidade funcional e o equilíbrio, com a finalidade de entender a importância da atividade física regular no comportamento motor de pacientes com DP. Além de estudos que buscam comparar grupos de pacientes com DP e idosos neurologicamente sadios.

\section{Contribuiç̧ão dos autores}

Paulo Cezar Rocha dos Santos: Concepção e projeto do artigo, tabulação, análise dos dados e interpretação dos dados, redação do texto. Luana Carolina de Morais: auxílio no desenvolvimento do projeto, na tabulação e na análise dos dados e na redação do artigo. Lucas Simieli: avaliação dos pacientes em relação à mobi- 
lidade, auxílio no desenvolvimento da redação e revisão crítica. Ellen Lirani-Silva: avaliação dos pacientes em relação aos parâmetros clínicos e ao equilíbrio e dados, revisão crítica da redação e auxílio na interpretação dos dados. Rodrigo Vitório: Avaliação dos pacientes em relação ao nível de atividade física, auxílio no desenvolvimento da redação e auxílio na interpretação dos dados. Maria Dilailça Trigueiro de Oliveira Ferreira: avaliação dos pacientes em relação à mobilidade e nível de atividade física e auxílio na tabulação e interpretação dos dados. Maria Joana Duarte Caetano: avaliação dos pacientes em relação à mobilidade e nível de atividade física e auxílio na tabulação e interpretação dos dados. Lilian Teresa Bucken Gobbi: auxílio no desenvolvimento do projeto, revisão crítica em relação à redação do texto.

\section{Referências}

1. Obeso JÁ, Rodriguez-Oroz MC, Rodriguez M, Lanciego, JL, Artieda J, Gonzalo N, Olanow CW. Pathophysiology of the basal ganglia in Parkinson's disease. Trends Neurosci. 2000;23:S8-S19.

2. Christofoletti G, Oliani M, Gobbi L, Gobbi S, \& Stella, F. Risk of falls among elderly people with Parkinson's disease and Alzheimer's dementia: A crosssectional study. Braz J Phys Ther. 2006;10:429-433.

3. Takakusaki K, Tomita N, \& Yano M. Substrates for normal gait and pathophysiology of gait disturbances with respect to the basal ganglia dysfunction. J Neurol. 2008;55(4):1929.

4. Grimbergen Y, Munneke M, \& Bloem B. Falls in Parkinson's disease. Curr Opin Neurol. 2004; 17:405-415.

5. Zampieri C, Salarian A, Carlson-Kuhta P, Nutt JG, Horak FB. Assessing mobility at home in people with early Parkinson's disease using an instrumented Timed Up and Go test. Parkinsonism Relat Disord. 2011;17:277-280.

6. Ashburn A, Stack E, Ballinger C, Fazakarley L, Fitton C. The circumstances of falls among people with Parkinson's disease and the use of Falls Diaries to facilitate reporting. Disabil Rehabil. 2008;30(16):1205-12.

7. Duncan RP, Leddy AL, Earhart GM. Management of balance and gait in older individuals with Parkinson's disease. Aging Health. 2011;7(2):205-218.

8. Gobbi LTB, Oliveira-Ferreira MDT, Caetano MJD, Lirani-Silva E, Barbieri FA, Stella F, Gobbi S. Exercise programs improve mobility and balance in people with Parkinson's disease. Parkinsonism Relat Disord. 2009;15(S3):S49-S52.

9. Podsiadlo D, Richardson S. The Timed "Up \& Go": A test of basic functional mobility for frail elderly persons. J Am Geriatr Soc. 1991;39:142-8.

10. Verheyden G, Kampshoff CS, Burnett ME, Cashell J, Martinelli L, Nicholas A, Stack EL, Ashburn A. Psychometric Properties of 3 Functional Mobility Tests for People With Parkinson Disease. Phys Ther. 2014 Feb;94(2):230-9

11. Miyamoto ST, Lombardi IJ, Berg KO, Ramos LR, Natour J. Brazilian version of the Berg Balance Scale. Braz J Med Biol Res. 2004;37:1411-1421.

12. Morris S, Morris, ME, Iansek R. Reliability of Measurements Obtained With the Timed "Up \& Go" Test in People With Parkinson Disease. Phys Ther. 2001;81(2):810-8.

13. Qutubuddin AA, Pegg PO, Cifu DX, Brown R, McNamee S, Carne W. Validating the Berg Balance Scale for patients with Parkinson's disease: a key to rehabilitation evaluation. Arch Phys Med Rehabil. 2005;86(4):789-92.

14. Horak F, Nutt J, Nashner L. Postural inflexibility in Parkinsonian subjects. J Neurol Sci. 1992;111:46-58.

15. Marsden CD, Parkes JD. Success and problems of long-term levodopa therapy in Parkinson's disease. Lancet. 1977;1:345-9.

16. Crizzle AM, Newhouse IJ. Is physical exercise beneficial for persons with Parkinson's disease? Clin J Sport Med. 2006;16:422-5.

17. Brusse KJ, Zimdars S, Zalewski KR, Steffen TM. Testing Functional Performance in People With Parkinson Disease. Research Report. Physical Tehrapy. 2005;85(2): 134-141.

18. Morris ME, Iansek R, Kirkwood B. A randomized control trial of movement strategies compared with exercise for people with Parkinson's disease. Mov Disord. 2009;24:64-71.

19. Falvo MJ, Schilling BK, Earhart GM. Parkinson's disease and resistive exercise: rationale, review and recommendations. Mov Disord. 2008;23:1-11. 
20. Voorrips LE, Ravelli AC, Dongelmans PC, Deurenberg P, Van Staveren WA. A physical activity questionnaire for the elderly. Med Sci Sports Exerc. 1991;23(8):974-979.

21. Fahn S, Elton R. Members of the UPDRS Development Comitee. The Unified Parkinson's Disease Rating Scale. In: Fahn S, Marsden CD, Calne DB, Goldstein M, editors, Recent developments in Parkinson's disease. Florham Park NJ: Mcmellam Health Care Information. 1987;2:153-63.

22. Hoehn MM, Yahr MD. Parkinsonism: onset, progression and mortality. Neurol. 1967; 17:573-81.

23. Almeida, O. Mini exame do estado mental e o diagnóstico de demência no Brasil. Arq Neuropsiquiatr. 1998;56:605-612.

24. Daly RM, Ahlborg HG, Ringsberg K, Gardsell P, Sernbo I, Karlsson MK.. Association between changes in habitual physical activity and changes in bone density, muscle strength, and functional performance in elderly men and women. J Am Geriatr Soc. 2008;56(12):2252-60.

25. Gonzaga JM, Barros SEB, Lisboa MGC, Barbieri FA, Gobbi LTB. Efeitos de diferentes tipos de exercício nos parâmetros do andar de idosas. Rev Bras Med Esporte. 2011;17(2): 166-170

26. Nelson ME, Rejeski WJ, Blair SN, Duncan PW, Judge JO, King AC, Macera CA, CastanedaSceppa C. Physical Activity and Public Health in Older Adults: Recommendation from the American College of Sports Medicine and the American Heart Association. Med Sci Sports Exerc. 2007;39:1435-1445.

27. Berg KO, Wood-Dauphinée SL, Williams JI, Maki B. Measuring balance in the elderly: validation of an instrument. Can J Public Health. 1992;83:S7-S11.

28. Berg KO, Maki BE, Williams JL, Holliday PJ, Wood-Dauphine SL. Clinical and laboratory measures of postural balance in an elderly population. Arch Phys Med Rehabil. 1992;73:1073-1080.

29. Salarian A, Horak FB, Zampieri C, Carlson-Kuhta P, Nutt JG, Kamiar A. iTUG, a sensitive and reliable measure of mobility. IEEE Trans Neural Syst Rehabil Eng. 2010;18(3):303-10.

30. Tajiri N, Yasuhara T, Shingo T, et al. Exercise exerts neuroprotective effects on Parkinson's disease model of rats. Brain Research. Jan 15 2010;1310:200-207.

31. Petzinger G, Fisher B, Van Leewmen J, Vucovik M, Akopian G, Meshul C, Holschneider D, Nacca A, Walsh J, \& Jakowec M. Enhancing neuroplasticity in the basal ganglia: the role of exercise in Parkinson's disease. Mov Disord. 2010;25:S141-S145.

32. Zigmond M, Cameron J, Leak R, Mirnics K, Russell V, Smeyne R, \& Smith A. Triggering endogenous neuroprotective processes through exercise in models of dopamine deficiency. Parkinsonism Relat Disord. 2009;15(S3):S42-S45.

33. Thompson M, Medley A. Performance of individuals with Parkinson's disease on the Timed up \& go. Neurol Rep. 1998;22(1):19-21.

\section{ENDEREÇO PARA}

CORRESPONDÊNCIA

PAULO CEZAR ROCHA DOS SANTOS

paulocezarr@hotmail.com
Rua 11-B, 608. Vila Indaiá, Rio Claro/SP -

Brasil. Cep: $13506-745$

Tel: (19) 3526-4365

Cel: (19) 98171-5558
RECEBIDO 08/01/2016

REVISADO 03/06/2016

APROVADO 28/06/2016 